\title{
Tumor-Associated Macrophages in Glioma: Friend or Foe?
}

\author{
Benjamin C. Kennedy, ${ }^{1}$ Christopher R. Showers, ${ }^{1}$ David E. Anderson, ${ }^{2}$ \\ Lisa Anderson, ${ }^{2}$ Peter Canoll, ${ }^{1}$ Jeffrey N. Bruce, ${ }^{1}$ and Richard C. E. Anderson ${ }^{1}$ \\ ${ }^{1}$ The Gabriele Bartoli Brain Tumor Research Laboratory, Department of Neurological Surgery, The Neurological Institute, \\ Columbia University College of Physicians and Surgeons, New York City, NY 10032, USA \\ ${ }^{2}$ Erinyes Biotechnologies LLC, Boston, MA 02118, USA
}

Correspondence should be addressed to Benjamin C. Kennedy; benjamin.c.kennedy@gmail.com

Received 6 November 2012; Accepted 8 April 2013

Academic Editor: Akira Hara

Copyright (c) 2013 Benjamin C. Kennedy et al. This is an open access article distributed under the Creative Commons Attribution License, which permits unrestricted use, distribution, and reproduction in any medium, provided the original work is properly cited.

\begin{abstract}
Tumor-associated macrophages (TAMs) contribute substantially to the tumor mass of gliomas and have been shown to play a major role in the creation of a tumor microenvironment that promotes tumor progression. Shortcomings of attempts at antiglioma immunotherapy may result from a failure to adequately address these effects. Emerging evidence supports an independent categorization of glioma TAMs as alternatively activated M2-type macrophages, in contrast to classically activated proinflammatory M1type macrophages. These M2-type macrophages exert glioma-supportive effects through reduced anti-tumor functions, increased expression of immunosuppressive mediators, and nonimmune tumor promotion through expression of trophic and invasionfacilitating substances. Much of our work has demonstrated these features of glioma TAMs, and together with the supporting literature will be reviewed here. Additionally, the dynamics of glioma cell-TAM interaction over the course of tumor development remain poorly understood; our efforts to elucidate glioma cell-TAM dynamics are summarized. Finally, the molecular pathways which underlie M2-type TAM polarization and gene expression similarly require further investigation, and may present the most potent targets for immunotherapeutic intervention. Highlighting recent evidence implicating the transcription factor STAT3 in immunosuppressive tumorigenic glioma TAMs, we advocate for gene array-based approaches to identify yet unappreciated expression regulators and effector molecules important to M2-type glioma TAMs polarization and function within the glioma tumor microenvironment.
\end{abstract}

\section{Introduction}

Malignant glioma is uniformly fatal with a median survival of less than 15 months with aggressive treatment [1]. Advances in surgical, radiation, and conventional chemotherapeutic therapies have had minimal impact on the prognosis of this aggressive disease [1]. The recalcitrance of malignant glioma to standard therapies is believed to result from phenotypic heterogeneity and diffuse infiltration into normal brain parenchyma [2], as well as residence within the unique immune environment of the central nervous system (CNS) [3]. Long viewed as an "immune-privileged" site due to a perceived lack of specialized antigen presenting cells (APCs), restriction from circulating lymphocytes and other immune mediators by the blood brain barrier (BBB), and absence of lymphatic drainage [4], the CNS appeared to possess little immunologic potential to resist glioma progression. Evidence accumulated over the last 20 years, however, has largely debunked this view of the CNS by demonstrating distinct immune activation cascades within the CNS in response to cerebral ischemia and traumatic brain injury $[5,6]$, contingent upon activation of resident microglia and infiltrating macrophages capable of effective antigen presentation and lymphocyte activation [68 ], all permissible through inducible permeability of the $\mathrm{BBB}$ to leukocytes and immune mediators in pathological states [9]. Activated microglia have been shown to express phenotypic and functional characteristics of both macrophages and dendritic cells [10], and furthermore are capable of inducing T-cell responses in a mixed lymphocyte-type reaction in vitro [11]. Additionally, circulating tumor-specific antibodies and cytotoxic T lymphocytes (CTL) have been isolated from the peripheral blood of patients with malignant glioma [12], 
indicating the potential for a competent tumoricidal immune response to glioma within the CNS. This expanding appreciation of intrinsic CNS immune capacity against glioma, coupled with the limited efficacy and profound side effects of current glioma therapies, has prompted a major investigation into immunotherapy as a therapeutic strategy against glioma.

The immune response to tumor-associated antigens and mechanisms of immunosuppression by tumor cells have been the most actively investigated areas of cancer immunotherapy research. These efforts have led to FDA-approved immunotherapy-based treatment protocols that have been shown to reduce tumor burden and prolong survival in patients with many different systemic tumors [13] including advanced prostate cancer and late stage melanoma [14, 15]. Unfortunately, immunotherapy against malignant glioma has so far met with only limited success [16]. At this time, the majority of immunotherapeutic efforts against malignant gliomas have focused on methods to try to stimulate an effective adaptive $\mathrm{T}$-cell response against glioma tumor antigens. This is largely because there is ample evidence demonstrating lymphocyte invasion into glioma tissue [17] and because studies have shown successful activation of a cytotoxic T-cell response using dendritic cell-based stimulation by glioma-specific antigens [16]. It is likely that current shortcomings of antiglioma immunotherapy are at least in part due to a failure to adequately address glioma-induced immunosuppression in the local tumor microenvironment [18]. Accordingly, reinvigorated attention has turned to the mechanisms by which glioma cells utilize immune mediators to alter immune behavior.

We and others have shown that tumor-associated monocytes and microglia (TAMs) are the predominant infiltrating immune cell in malignant glioma and can account for up to $40 \%$ of the tumor cell mass [19-21]. Because the frequency of TAMs greatly outnumbers lymphocytes in human gliomas, it is possible that TAMs, under the influence of glioma cells, are playing a major role in the creation of a local tumor microenvironment that is immunosuppressive and promotes glioma growth [22-28]. Considerable efforts to phenotypically and functionally characterize glioma TAMs have led to a delineation between classically activated inflammatory M1type macrophages with tumoricidal potential from immunosuppressive M2-type macrophages, thought to predominate in the glioma microenvironment [29].

Classically activated M1-type macrophages participate in the coordinated response to immunogenic antigens primarily through production of proinflammatory mediators (such as TNF- $\alpha$, IL-1B, and IL-12), upregulation of cell surface molecules necessary for antigen presentation (including MHC II and costimulatory molecules CD80 and CD86), and an overall enhanced ability to phagocytose pathogenic material [30, 31]. Conversely, alternatively activated M2-type macrophages do not secrete the proinflammatory mediators IL-1B or TNF- $\alpha$ [32] and are believed to exert immunomodulation primarily through secretion of the potent immunosuppressive cytokines IL-10, IL-6, and TGF- $\beta$, downregulation of cell surface molecules necessary for antigen presentation including MHC II, CD80, and CD86, decreased phagocytic capacity, and upregulation of cell surface antigens FasL and
B7-H1 both known to stimulate programmed cell death in lymphocytes, among other effects $[29,33,34]$. See Figure 1 for a summary of the M1 and M2 macrophage phenotypes in glioma.

Recent refinements of this characterization scheme describe a more heterogeneous population of myeloid-derived cells at different stages of maturation, able to suppress multiple phases of the immune response [35]. Termed myeloidderived suppressor cells (MDSC), cells of this expanded immunosuppressive category have been shown to both perpetuate the glioma-promoting microenvironment as well as distribute peripherally to hinder lymphocyte activation in immune organs [33]. Still, the underlying cellular mechanisms and glioma-TAM interactions dictating the immunomodulatory function of TAMs in glioma remain unclear despite some evidence describing aspects of a complex network of autocrine and paracrine loops of cytokine and chemokine signaling. Is the glioma-promoting relationship [36] between TAMs and the tumor cells present at tumor initiation, or do glioma cells reeducate classically activated TAMs to express an alternative MDSC phenotype at some point in tumor progression? What intrinsic signaling motifs or master regulators of gene expression underlie immunosuppressive TAM phenotypes under the influence of the complex tumor microenvironment? Do certain convergent effector molecules/pathways within TAMs exert disproportionate effects on immunosuppression or glioma facilitation? In this review we will highlight efforts directed at these questions, as their answers may provide information crucial to the development of effective clinical immunotherapy against malignant gliomas.

\section{Origins of Tumor-Associated Macrophages in Glioma}

Different mononuclear cell-derived populations of distinct lineages exist within the central nervous system (CNS) under pathological conditions. TAMs in human glioma are generally believed to originate from at least two distinct sources. Principal among them are resident microglia, believed to monitor their local neural tissue environment through extensive ramifications, and subsequently to activate a phagocytic phenotype, nearly identical to activated macrophage phenotypes, upon stimulation [37]. A recent fate mapping analysis demonstrated that resident microglia are a distinct lineage that arise from embryonic yolk sac myelomonocytes, which populate the primitive CNS prior to definitive hematopoiesis [38]. Clear evidence has established that activated resident microglia form a large component of macrophages within glioma tissue $[19,39,40]$. A second group of immune cell macrophage precursors in the $\mathrm{CNS}$ are peripheral bone marrow-derived mononuclear cells, which colonize the CNS under pathological conditions. Recruitment, engraftment, and subsequent macrophage activation of peripheral mononuclear cells have been established in many experimental models of CNS disease [41-45] and were recently demonstrated to contribute significantly to the macrophage content of human gliomas $[19,46]$. Differentiating the lineage origin of individual TAMs isolated from human glioma tissue has 


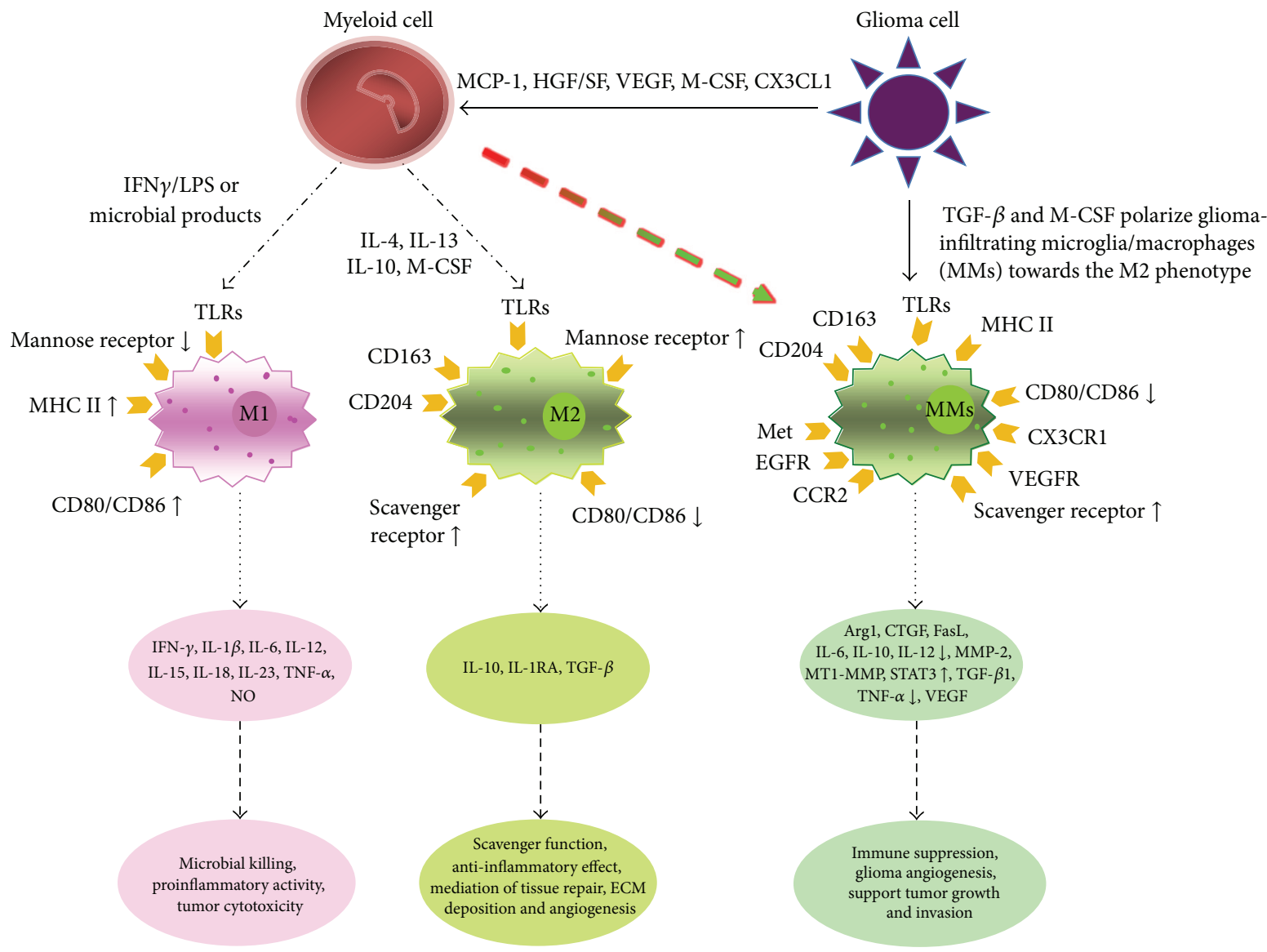

Figure 1: Microglia in glioma are polarized. M1 (classically activated macrophages) and M2 (alternatively activated macrophages) differ with respect to activating signals, receptor expression, cytokine/chemokine production, and biological behavior. When mononuclear/phagocytic cells are stimulated by IFN- $\gamma$ lipopolysaccharides and other microbial products, they differentiate into the M1 phenotype. Microbial products are recognized by pattern recognition receptors (PRRs) on the surface of M1, such as TLRs, and stimulate the production of pro-inflammatory cytokines as well as the expression of receptors that are involved in antigen presentation. When mononuclear/phagocytic cells are activated by IL-4, IL-13, IL-10, and M-CSF, they differentiate into the M2 phenotype. Tumor-derived molecules, such as TGF- $\beta$ and M-CSF, can polarize glioma-infiltrating microglia/microphages (MMs) toward the M2 phenotype and accordingly stimulate the production of anti-inflammatory molecules. Some other glioma-derived molecules, such as MCP-1 and VEGF, can recruit myeloid cells into the tumor site. Published with permission from Li and Graeber [29].

proven to be difficult. Most attempts have used FACS sorting of ex vivo specimens based on differential levels of CD45 expression in cells coexpressing CD11b, a technique validated in glioma homogenates of chimeric rats [19]. Still, phenotypic and functional differences between these constituent groups of TAMs in gliomas remains largely unknown.

\section{Features of Tumor-Associated Macrophages in Glioma}

As previously mentioned, the overwhelming predominance of TAMs in the immune infiltrate of both murine and human malignant gliomas has heightened awareness of the influential role these cells may have on both creation of an immunosuppressive tumor microenvironment and facilitation of glioma cell progression [21, 47]. Cumulative research suggests that TAMs within malignant gliomas are dominated by the immunosuppressive M2-type subtype, as the following characteristics have been shown: (1) deficiencies in expected antitumor effector functions of classically activated M1-type macrophages, (2) expression of multiple immunosuppressive antigens and soluble mediators hindering a multifaceted antitumor immune response to glioma tissue, and (3) expression of multiple glioma-promoting mediators including tumor growth and angiogenic factors in addition to stromal remodeling agents, altogether augmenting glioma progression.

3.1. Reduced Antitumor Function in Glioma TAM. Despite clear evidence of chemotaxis to glioma tumor tissue and subsequent contact with glioma-specific antigens known to be classically immunogenic [18], TAMs in malignant gliomas demonstrate a significant reduction in specific proinflammatory or antitumor effects. Much of this is evidenced by studies showing reductions in secretion of proinflammatory cytokines and increases in secretion of inhibitory cytokines. For instance, our group recently reported that in the presence 
of malignant glioma cells, there is nearly complete abrogation of TNF- $\alpha$ and a significant upregulation of IL-10 secretion by stimulated naïve human monocytes in vitro [20]. These findings were subsequently recapitulated in vivo when we used our murine glioma model to demonstrate a reduction of TNF- $\alpha$ expression by TAMs during late stages of tumor growth [21]. Figure 2 illustrates these findings.

The mature M1 macrophage marker CD14 serves as a coreceptor of TLR4 and is upregulated in nearly all CNS pathologies [48]. However, downregulation of CD14 has been observed in TAMs in several other cancers, and Rodriguez and Parney et al. demonstrated that monocytes isolated from healthy subjects dramatically reduce expression of CD14 but not CD11b upon exposure to human glioma cell lines [49]. This represents another potential mechanism by which TAMs have diminished antiglioma activity.

Other deficiencies of glioma TAMs thought to contribute to local immunosuppression are reduction in the expression of HLA and costimulatory molecules. For instance, our group has previously shown significantly reduced CD80 and HLADR expression on stimulated naïve human monocytyes when cocultured with GBM cells [20]. This finding is consistent with that of Badie and Schartner who used FACS to demonstrate little to no expression of MHCII, CD80, or CD86 on macrophages freshly isolated from rat gliomas [19]. Furthermore, expression of these costimulatory molecules on TAMs could not be restored by stimulation with IFN- $\gamma$ or LPS [50]. These phenotypic changes on TAMs are likely to be functionally significant in vivo, as we have found that monocytes reisolated following coculture with malignant glioma cells demonstrate an inability to activate allogenic $\mathrm{CD} 4^{+} \mathrm{T}$ cells [20]. In addition, there is suppressed secretion of IFN$\gamma$ from $\mathrm{CD}^{+}{ }^{+} \mathrm{T}$ cells cultured with GBM-treated monocytes [20]. Similar results regarding downregulation of cell surface molecules and absence of T-cell activation were reported by Rodrigues et al., as well as further demonstrating that, following coculture with malignant glioma cells, human monocytes induce apoptosis in activated autologous T cells [49], a known outcome of incomplete macrophage-T cell-communication.

Glioma TAMs have also been shown to be deficient in phagocytosis. Rodrigues and colleagues demonstrated a significant reduction in the ability to phagocytose bacterial cell wall particles following stimulation in glioma cellconditioned monocytes, as compared with both astrocyteconditioned and unconditioned monocytes [49]. In another report, Hussain et al. demonstrated active phagocytosis of opsonized beads in macrophages isolated from ex vivo human GBM specimens [32]. This group further attempted to show that TAMs isolated directly from human GBM tumors are deficient of non-MHC-restricted antitumor cellular toxicity through coculture with a target cell line derived from malignant human glioma. Their results indicate minimal cytotoxic ability of these glioma TAMs, as compared to naïve microglia isolated from normal brain tissue [32].

3.2. TAM-Mediated Immunosuppression in Glioma. In addition to the antitumor effector function deficiencies described earlier, accumulating evidence suggests that glioma TAMs are actively immunosuppressive. Glioma TAMs are now thought to represent an altered phenotype resulting from the directed influence of tumor cells upon immune cells in efforts to produce a favorable tumor microenvironment. Among the immunosuppressive mediators upregulated in glioma TAMs, those that appear to exert a predominate effect include cytokines IL-10, TGF- $\beta$, and cell surface antigens B7-H1, and FasL [20, 29, 33, 34]. Malignant glioma cells have also been shown to induce tolerance.

Our group has previously demonstrated that when cocultured with malignant glioma cells, stimulated naïve human monocytes significantly upregulate expression of IL-10 [20]. Quantitative PCR analysis of these monocytes re-isolated following co-culture demonstrated upregulation of STAT3 as well. Similar results were seen when TAMs from human malignant gliomas isolated directly ex vivo were compared with TAMs from meningiomas and these findings are presented in Figure 3. Activation of the transcription factor STAT3 in both glioma cells and glioma TAMs has been suggested to be a key intracellular mediator coordinating the expression of these immunosuppressive molecules $[48,51]$, leading Brantley and Benveniste to describe STAT3 as a critical "molecular hub" linking multiple pathways in glioma biology [52]. These findings are consistent with earlier work of Wagner et al. who, using multiple molecular techniques, localized both gene expression and IL-10 protein molecules in ex vivo GBM tumor specimens overwhelmingly to TAMs, though also present in glioma cells to a much lesser extent [27]. Furthermore, in a recent series of similar experiments, monocytes isolated from healthy subject dramatically increased expression of both IL-10 and TGF- $\beta$ following coculture with glioma cell lines, as compared to isolated culture as well as co-culture with NHA [49]. Hence, expression of IL-10 by TAMs in glioma tissue appears to be an important immunosuppressive mediator of the glioma microenvironment, and its concomitant expression in glioma cells may serve both as an initial chemotactic agent to recruit monocytes, as well as the driver of feedforward loops of immunosuppressive mediator expression between tumor cells and macrophages within the tumor microenvironment $[29,34]$.

Both glioma cells [53] and TAMs have been shown to express the cell death pathway molecule FasL; indeed Badie et al. demonstrated that nearly every infiltrating monocytederived cell in murine glioma models expressed FasL [54]. Furthermore, evidence that apoptotic lymphocytes in the GBM microenvironment express Fas [55] and that microglia induce the apoptotic death of activated T lymphocytes in coculture [56] together promoted the theory that TAMs may directly exert an immunosuppressive death signal to gliomainfiltrating lymphocytes. This hypothesis is strengthened by the finding that neutralization of FasL results in a significant increase in the number of tumor-infiltrating lymphocytes in a murine glioma model [57]. Other reports, however, have shown that TAMs isolated directly from human glioma resection tissue stained at very low levels or not at all for FasL, leading the authors to conclude that FasL-Fas mediated apoptosis is not a predominant mechanism of immune evasion by TAM in human glioma [32]. Although the precise role of FasL expression on the surface of TAMs within the glioma microenvironment remains unclear, further studies 


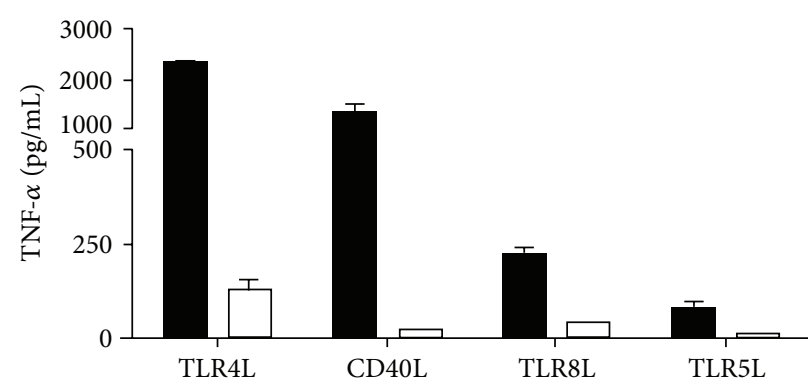

Mo alone

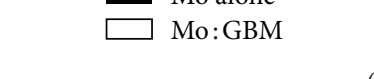

(a)

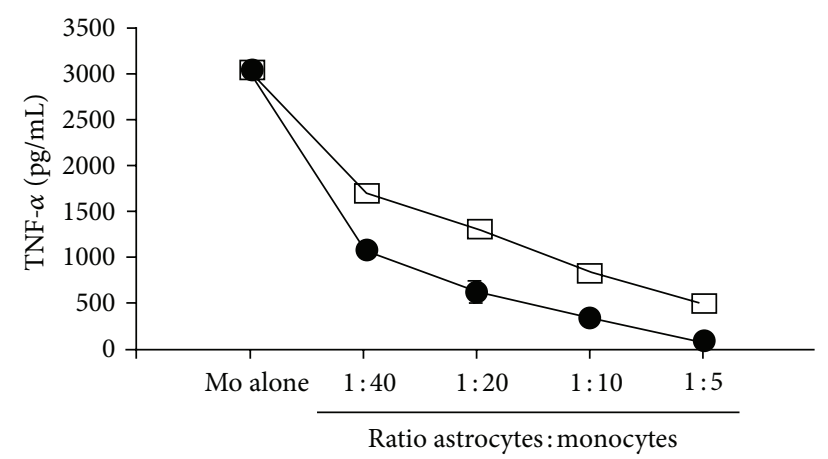

Mo: GBM

$\exists$ Mo:NHA

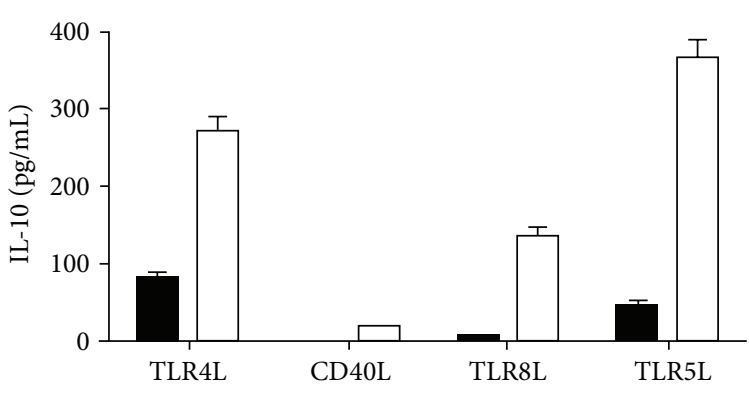

Mo alone Mo:GBM

(b)

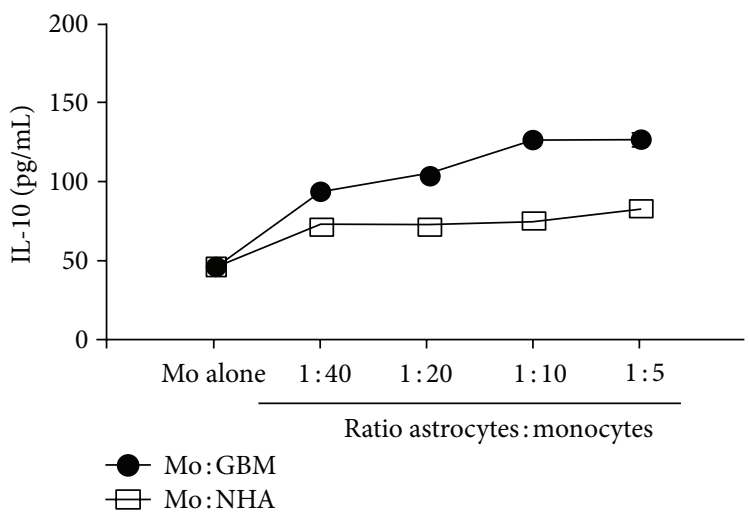

(d)

(c)

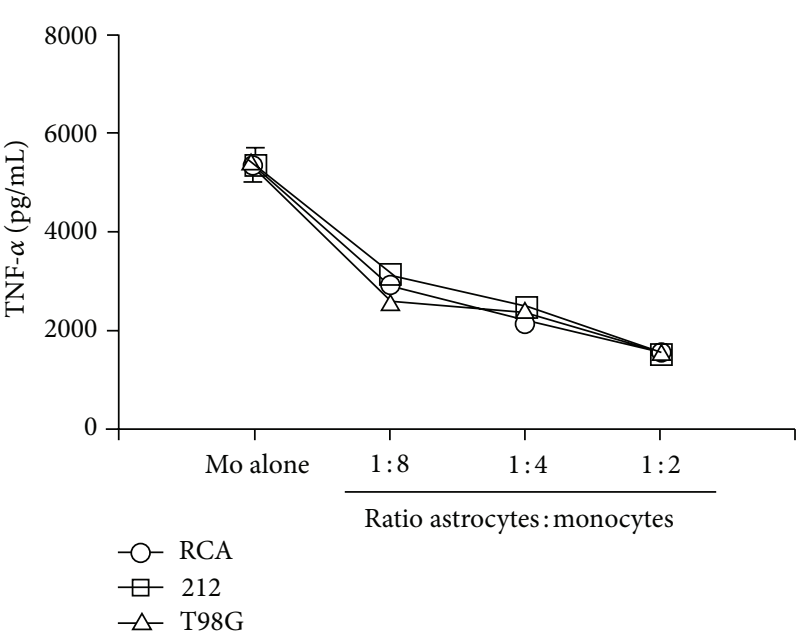

(e)

FIGURE 2: GBM tumor cells suppress monocyte (Mo) activation by a variety of stimuli. ((a) and (b)) Ex vivo monocytes were stimulated with the indicated stimuli in the absence or presence of GBM tumor cells, and TNF- $\alpha$ and IL-10 secretion was measured after $48 \mathrm{~h}$. Comparable results were seen in five independent experiments. ((c) and (d)) Ex vivo monocytes were stimulated with LPS (1 g/mL) in the presence of the indicated ratios of monocytes:GBM tumor cells or NHA. Comparable results were seen in two independent experiments. (e) Ex vivo monocytes were stimulated with LPS $(1 \mathrm{~g} / \mathrm{mL})$ in the presence of the indicated ratios of monocytes and two primary GBM cell lines (RCA and 212) and an extensively passaged GBM cell line (T98G). Comparable results were seen in two independent experiments. SD is represented in all cases. Unstimulated monocytes were used in all assays, and secretion of both TNF- $\alpha$ and IL-10 was below the limit of detection. Publised with permission from Kostianovsky et al. [20]. 


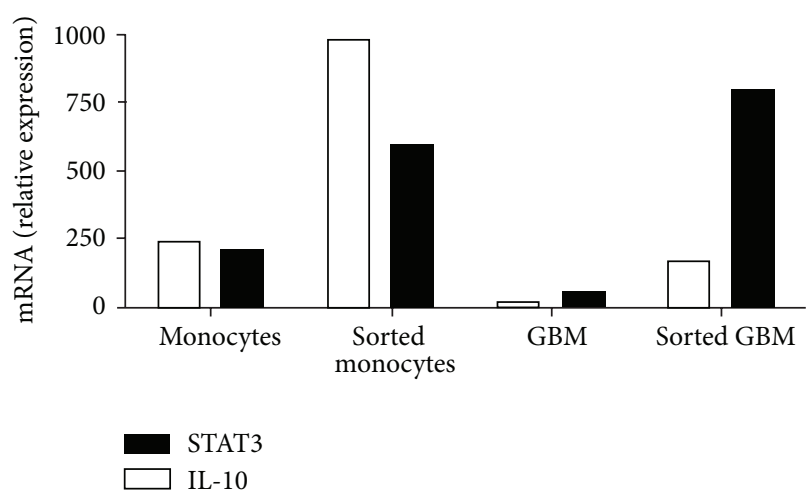

(a)

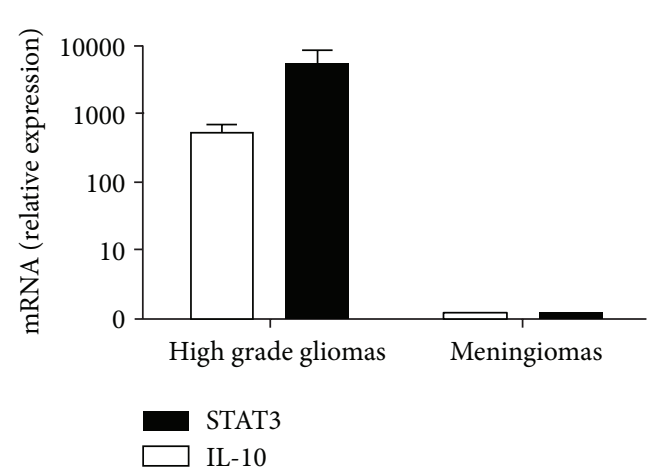

(b)

FIGURE 3: Upregulation of STAT3 and IL-10 in monocytes occurs after coculture with GBM tumor cells in vitro and ex vivo. (a), ex vivo monocytes were stimulated with LPS in the absence or presence of GBM tumor cells for $4 \mathrm{~h}$, at which point monocytes and GBM tumor cells were isolated by FACS. RNA was isolated and levels of IL-10 and STAT3 were measured by quantitative RT-PCR. Similar results were seen in

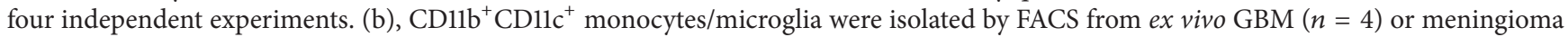
$(n=2)$ tumor specimens and RNA was isolated and analyzed for expression of IL-10 and STAT3 by quantitative RT-PCR. Published with permission from Kostianovsky et al. [20].

investigating its role in glioma-induced immunosuppression are clearly indicated.

B7-homolog 1 (B7-H1) is a homolog of the costimulatory surface antigens CD80 and CD86 (B7.1 and B7.2) that has been shown to attenuate $\mathrm{T}$-cell receptor function through engagement of the programmed death receptor (PD-1) on the surface of T cells [18]. PD-1 activation on T cells by B7-H1 has been shown to initiate an intracellular signaling cascade resulting in downregulation of T-cell receptor (TCR) signaling [58] and may also promote T-cell apoptosis [63]. Parsa et al. and Wintterle et al. have confirmed near ubiquitous expression of $\mathrm{B} 7-\mathrm{H} 1$ on glioma cells $[58,59]$, and Rodrigues and colleagues have recently shown $\mathrm{B} 7-\mathrm{H} 1$ expression on human macrophages following co-culture with allogeneic glioma cell lines [49]. Though the precise expression pattern and role of B7-H1 remain unclear, mutual expression of B7-H1 in both glioma and TAM cells may prove a critical mechanism by which lymphocyte suppression is achieved in the tumor microenvironment.

Although the mechanisms are unknown, there is evidence that malignant gliomas are able to alter monocytes so they become tolerogenic. When human monocytes previously cultured with malignant glioma cells are co-cultured with naïve monocytes, naive monocytes had a dramatically reduced ability to secrete TNF- $\alpha$ in response to stimulation [20]. These findings suggest that inhibition of classically activated antitumor effector functions of glioma TAMs may be a long-lasting regulatory phenotype.

3.3. Nonimmune Glioma Promotion. Glioma cells are known to produce a number of self-supportive factors concurrently with their corresponding cell surface receptors, together acting to promote their own proliferation, migration, angiogenesis, and subsequently tumor extension [60-62]. Indeed, current models of the glioma tumor microenvironment suggest a potent milieu of trophic and immunomodulatory factors bathing all tumor cells and propagating tumor growth through autocrine and paracrine loops of expression and stimulation [29]. Less clear than their lack of effector function or their expression of immunosuppressive mediators, glioma TAMs are increasingly implicated in the contribution of glioma-promoting tumor trophic factors to the local microenvironment. Among the tumor supportive factors potentially secreted by TAMs, TGF- $\beta$, EGF, and HGF/SF have drawn the most attention, though dissecting the precise role of TAMs in the production of these trophic factors remains to be accomplished.

Tissue growth factor beta (TGF- $\beta$ ) is an extremely potent immunosuppressive and transformative cytokine whose expression is mostly associated with glioma cells themselves $[63,64]$ and is believed to have a major influence in directing the alternatively activated immunosuppressive phenotype of TAMs [65]. In addition to the well-documented immunomodulatory and tumor cell tropic effects of TGF$\beta$ through induction of VEGF and FGFs [66], TGF- $\beta$ may also promote tumor cell migration and invasion through induction of MMP expression in conjunction with suppression of tissue inhibitor of metalloproatease expression [67], together affecting stromal remodeling to facilitate invasion. Microglia have been shown to produce TGF- $\beta$ isoform 1 (TGF- $\beta 1$ ) under certain pathological conditions including neuritis and trauma $[68,69]$. Using in situ hybridization, Kiefer et al. localized the expression of the TGF- $\beta 1$ isoform to activated glioma TAMs in a murine model, suggesting to the authors this isoform's involvement in a mutually reinforcing paracrine loop with glioma cells [70]. Building upon this hypothesis, Li and Graeber proposed that, whereas glioma-derived TGF- $\beta$ exerts immunosuppression by driving alternative polarization in TAMs, TGF- $\beta$ produced by the glioma TAMs may promote tumor growth and invasion by stimulating the upregulation of its own cognate receptors TBRI and TBRII on glioma cells [29] enabling a more potent trophic response to the high concentration of TGF- $\beta$ proposed to exist in the glioma microenvironment. 
Epidermal growth factor expression and stimulation of its cognate receptor (EGF/EGFR) have emerged as a pivotal signaling mechanism in high grade glioma. EGFR amplification is seen in approximately $50 \%$ of GBM, and in approximately $50 \%$ of those tumors the glioma cells express EGFRvIII, a mutant receptor that persistently activates downstream immunosuppressive pathways including those involving STAT3 [71]. In two separate efforts, activated microglia from a murine glioma model demonstrated expression of EGFR [72] as well as low levels of EGF secretion [73]. These initial findings again position TAMs within a potential paracrine network with glioma cells, acting to reinforce expression of both EGF and EGFR on glioma cells to promote tumor progression.

Hepatocyte growth factor/scatter factor acts exclusively through the tyrosine kinase receptor c-Met and expression of both the soluble ligand and receptor has been demonstrated in both ex vivo human glioma and TAM cells $[74,75]$. Kunkel et al. used combined in situ hybridization with fluorescence immunohistochemistry to demonstrate expression of both HGF/SF and c-Met in a majority of TAMs isolated from human ex vivo GBM specimens [75]. Badie et al. demonstrated in vitro that glioma-derived HGF/SF is a potent chemotactic agent on microglia [61] postulating that tumorsecreted HGF/SF acting upon TAM c-Met receptors may be a major mechanism by which glioma tissue recruits monocytes to commandeer toward the construction of a favorable microenvironment. Stimulation of c-Met by HGF/SF in human GBM cell lines has been shown to increase proliferation and invasive motility [74] and furthermore to induce angiogenesis in murine glioma tissues [76], yet it remains unclear if this latter effect is mediated through direct action on glioma endothelial cells or through induction of VEGF. Indeed, in separate efforts, radiation and hypoxia were shown to induce c-Met expression in glioma cells, further supporting its role in glioma tumor angiogenesis [77, 78]. Altogether these findings again suggest a mutually reinforcing network of HSF/SF upon c-Met paracrine signaling between glioma cells and TAMs, whereby glioma cells recruit monocytes in the alternatively activating tumor microenvironment to subsequently derive trophic stimulation by alternatively mature TAM secretion of HGF/SF.

\section{Dynamics of Glioma-Tam Interaction}

A major shortcoming of the efforts to understand gliomaassociated macrophages remains a paucity of data describing the dynamics of glioma cell and macrophage interactions. Most of the findings reviewed to this point are based upon ex vivo human samples taken at the time of surgical resection, or similarly upon murine specimens harvested at a late-stage time point when glioma tumor mass is grossly apparent. Both of these scenarios likely represent end-stage tumors wherein the tumor has advanced to aggressive behavior, abetted by immunosuppressive glioma-promoting TAMs, as this review has demonstrated. Little data is available to suggest if the partnership between glioma cells and alternatively activated M2 TAMs arises at tumor onset and therefore contributes early in tumor formation, or if TAMs initially

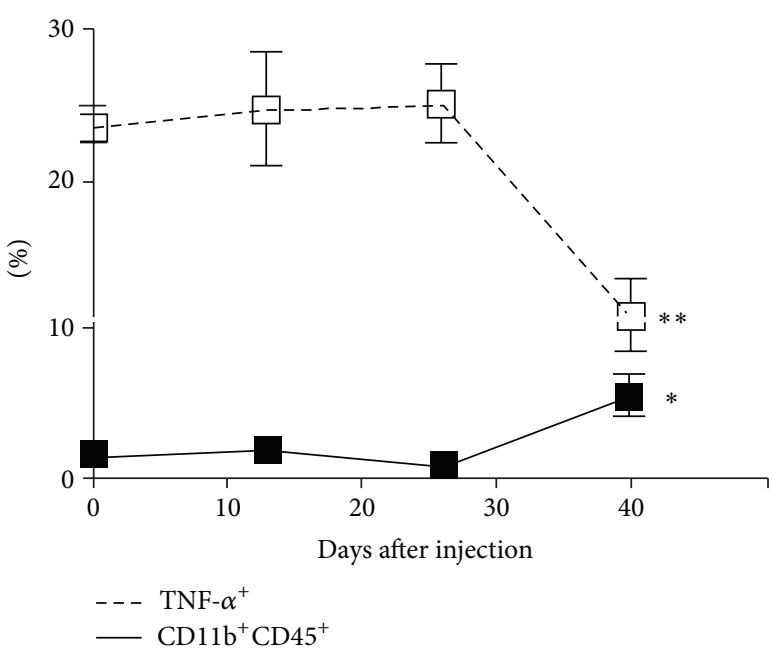

FIGURE 4: Frequency and functional changes in tumor-associated macrophages and microglia (TAMs) in ex vivo tumor specimens over the course of tumor development. While the percentage of TAMs $\left(\mathrm{CD} 11 \mathrm{~b}^{+} \mathrm{CD} 45^{+}\right.$cells) was increased by the final time point (1.1\% versus $5.6 \%, P=0.017$ ), their functional capacity was impaired as measured by TNF- $\alpha$ expression ( $25.2 \%$ versus $10.9 \%, P=0.007)$. Thirteen mice were analyzed at $13 \mathrm{dpi}(n=4), 26 \mathrm{dpi}(n=4)$, and at 29 dpi upon exhibiting clinical tumor morbidity $(n=1)$ or at $40 \mathrm{dpi}$ $(n=4)$. Differences between the means at each time point were tested using two-sided $t$-tests with unequal variances. ${ }^{*} P<0.05$, ${ }^{* *} P<0.01,{ }^{* * *} P<0.001$. Published with permission from Kennedy et al. [21].

manifest a classically activated M1 phenotype to combat tumor development until some critical point when tumorderived mediators overwhelmingly direct M2 polarization, followed by rapid tumor progression and clinical presentation. Recent efforts by our group have sought to address these questions through kinetic studies of the infiltration and function of immune cells in a murine glioma model, both within the tumor microenvironment and peripherally [21]. Using multiparameter FACS, we assessed TAM frequencies at early, intermediate, and late time points following injection of a PDGF-expressing retrovirus $(13,26$, and 40 days after injection) and demonstrated little change in TAM frequency between early and intermediate time points, despite a substantial increase in TAM frequency in the final time point. Furthermore, at each time point, TAM function status was assessed through evaluation of TNF- $\alpha$ secretion. Of great interest, no change was observed in the proportion of TAMs secreting TNF- $\alpha$ between the early and intermediate time points, though a 2.5 -fold reduction in the TNF- $\alpha$-secreting TAMs was evident by the final time point. These findings are summarized in Figure 4. Taken together in the context of findings referenced in this review, these observations may suggest a fulcrum in TAM activation status at some point between the intermediate and late time points, whereafter glioma cells are able to tip the balance toward an alternatively activated M2 TAM phenotype, thereby amplifying autocrine and paracrine loops successful in recruiting greater numbers of TAMs which do not express TNF- $\alpha$. 
Peripheral immune changes were investigated with splenic preparations, evaluated for presence of IFN- $\gamma$-producing $\mathrm{CD} 4^{+} \mathrm{T}$ cells and regulatory $\mathrm{T}$ cells $\left(\mathrm{CD} 4^{+} \mathrm{FoxP}^{+}\right)$. Between the early and intermediate time points, the proportion of IFN- $\gamma$-producing $\mathrm{CD}^{+}{ }^{+} \mathrm{T}$ cells, increased modestly and sustained this elevated frequency through the late time point. Interestingly, the proportion of regulatory $\mathrm{T}$ cells increased significantly between the early and intermediate time points and also remained elevated, though slightly decreased from peak frequency, through the late time point. Moreover, the proportion of these T-reg cells that expressed IL-10 mirror the overall frequency, increasing significantly between early and intermediate time points and exhibiting a sustained effect through the late time point. These findings suggest that peripheral immunosuppressive changes may precede those in the tumor microenvironment. Efforts of our group and others have established regulatory $\mathrm{T}$ cells and others as major immunosuppressive cellular mediators implicated both locally and systemically in glioma patients [79-81]. The crosstalk between glioma-associated TAMs and peripheral or glioma-infiltrating Tregs is likely complex, and our work may suggest that early Tregs may have a role in the induction of the later M2 TAM phenotype. Our group and others are continuing to pursue further characterization of these intercellular relationships, leading us toward a more nuanced understanding of the process of creating the immunosuppressive glioma microenvironment.

\section{Underlying Mechanisms of Alternatively Activated TAMs}

As this review suggests, the creation of a potent immunosuppressive, glioma conducive, tumor microenvironment at least in part results from the complex interplay of glioma cells and alternatively activated TAMs that involve many immune mediators with pleiotropic affects. While extensive efforts to characterize glioma TAMs have established many of the individual immunosuppressive and tumor permissive features specific to these cells, an understanding of the molecular pathways and signaling molecules affecting gene expression which lead to the altered phenotype of glioma TAMs is not known. Intracellular mediators at points of signaling convergence within glioma TAMs and the molecular products which result from their activation present potential targets for immunotherapy-based strategies directed against malignant gliomas. Furthermore, recent evidence highlighting prognostic differences among distinct molecular subtypes of glioma, including those with $1 \mathrm{p} / 19 \mathrm{q}$ codeletion, isocitrate dehydrogenase (IDH) mutations, and differential MGMT methylation status [82], raises the possibility of subtype specific differences in both glioma cell and TAM expression profiles, and therefore in the composition of the tumor microenvironment; such discrepancies remain to be explored.

At this point, recognition of differential activation of the signal transducer and activator of transcription 3 protein (STAT3) within both glioma cells [83] and glioma TAMs [84] is the most compelling evidence of a single gene involved with multiple immunosuppressive signaling pathways in glioma induced immunosuppression. STAT3 activation in glioma
TAMs is induced by many mediators known to compose the local tumor microenvironment such as IL-10, IL-6, EGF, and FGF [85]. Activated STAT3 is known to reduce the expression of surface molecules necessary for antigen presentation such as MHCII, CD80, and CD86 [86], as well as to increase the expression of many M2 specific immunomodulatory mediators including IL-10, EGF, VEGF, and various MMPs $[51,52]$. Therefore, STAT3 may serve as a critical "molecular hub" [52] linking multiple pathways unique to alternatively activated M2 type TAMs. Furthermore, STAT3 target molecules such as IL-10 and IL-6 have been shown to activate STAT3 [51], leading Li and Graeber to propose a feed-forward mechanism which may account for the constitutive activation of STAT3 in both glioma cells and glioma-infiltrating TAMs [29].

Though STAT3 activation appears to play a key role in generating and perpetuating the M2-type TAMs in gliomas, it is unclear whether a single dominant molecule or a complex network of molecules is responsible for the immunosuppressive phenotype of glioma TAMs. Our group therefore conducted a microarray-based approach of sorted human monocytes after co-culturing with malignant gliomas in an attempt to better characterize these pathways. Through extensive pathway analyses and network exploration, we identified a small subset of novel candidate genes that could be responsible for glioma-induced dysfunction of TAMs. To demonstrate that these candidate genes identified by the microarray analysis were important in vivo, we then isolated TAMs from patients with primary and recurrent malignant gliomas and confirmed their differential expression patterns ex vivo. Our next step is to use siRNA to block these candidate genes in our GBM: monocyte co-culture model to determine if monocyte function can be restored. Importantly, preliminary analysis confirms that STAT3 is not among our candidate genes, suggesting that multiple pathways contribute to TAM dysfunction in gliomas. We hope that the identification of genes differentially expressed in glioma TAMs may give us a better understanding of the pathways driving M2-type polarization and lead to new, more effective targets for glioma based immunotherapy.

\section{Conclusions}

Are tumor-associated monocytes/microglia in malignant gliomas friends or foes? Although a comprehensive answer to this question remains elusive, the considerable efforts described in this review seem to cast TAMs in glioma as a formidable foe, espousing an altered activation state within the local tumor microenvironment characterized by deficiencies in antitumor effector functions, upregulation of potent immunosuppressive mediators, and participation in tumorigenic loops of paracrine signaling involving expression of trophic factors and their cognate receptors. Although the dynamics of this malign partnership between glioma cells and TAMs remain unclear over the course of tumor progression, a turning point seems to occur late in tumor development, perhaps providing a better opportunity for clinically based immunotherapy. Given the compelling evidence that TAMs contribute significantly to the creation and maintenance of immunosuppression and tumor progression, it is unlikely 
that clinically effective immunotherapy against malignant gliomas will be achieved until we gain a better understanding of how to influence TAM function in the local tumor microenvironment.

\section{References}

[1] H. Ohgaki and P. Kleihues, "Epidemiology and etiology of gliomas," Acta Neuropathologica, vol. 109, no. 1, pp. 93-108, 2005.

[2] E. C. Holland, "Glioblastoma multiforme: the terminator," Proceedings of the National Academy of Sciences of the United States of America, vol. 97, no. 12, pp. 6242-6244, 2000.

[3] B. D. Choi, K. S. Chen, and J. H. Sampson, "Tumors of the central nervous system," in Tumors of the Central Nervous System, M. A. Hayat, Ed., vol. 1, Springer, Dordrecht, The Netherlands, 2011.

[4] Z. Fabry, C. S. Raine, and M. N. Hart, "Nervous tissue as an immune compartment: the dialect of the immune response in the CNS," Immunology Today, vol. 15, no. 5, pp. 218-224, 1994.

[5] C. J. Harling-Berg, J. J. Hallett, J. T. Park, and P. M. Knopf, "Hierarchy of immune responses to antigen in the normal brain," Current Topics in Microbiology and Immunology, vol. 265, pp. $1-22,2002$.

[6] J. Gehrmann, R. B. Banati, C. Wiessner, K. A. Hossmann, and G. W. Kreutzberg, "Reactive microglia in cerebral ischaemia: an early mediator of tissue damage?" Neuropathology and Applied Neurobiology, vol. 21, no. 4, pp. 277-289, 1995.

[7] W. F. Hickey and H. Kimura, "Perivascular microglial cells of the CNS are bone marrow-derived and present antigen in vivo," Science, vol. 239, no. 4837, pp. 290-292, 1988.

[8] R. Beschorner, T. D. Nguyen, F. Gözalan et al., "CD14 expression by activated parenchymal microglia/macrophages and infiltrating monocytes following human traumatic brain injury," Acta Neuropathologica, vol. 103, no. 6, pp. 541-549, 2002.

[9] T. Owens, T. Renno, V. Taupin, and M. Krakowski, "Inflammatory cytokines in the brain: does the CNS shape immune responses?" Immunology Today, vol. 15, no. 12, pp. 566-571, 1994.

[10] E. Ulvestad, K. Williams, R. Bjerkvig, K. Tiekotter, J. Antel, and R. Matre, "Human microglial cells have phenotypic and functional characteristics in common with both macrophages and dendritic antigen-presenting cells," Journal of Leukocyte Biology, vol. 56, no. 6, pp. 732-740, 1994.

[11] K. Williams Jr., E. Ulvestad, L. Cragg, M. Blain, and J. P. Antel, "Induction of primary T cell responses by human glial cells," Journal of Neuroscience Research, vol. 36, no. 4, pp. 382-390, 1993.

[12] S. Miescher, T. L. Whiteside, N. de Tribolet, and V. von Fliedner, "In situ characterization, clonogenic potential, and antitumor cytolytic activity of $\mathrm{T}$ lymphocytes infiltrating human brain cancers," Journal of Neurosurgery, vol. 68, no. 3, pp. 438-448, 1988.

[13] A. B. Heimberger and J. H. Sampson, "Immunotherapy coming of age: what will it take to make it standard of care for glioblastoma?" Neuro-Oncology, vol. 13, no. 1, pp. 3-13, 2011.

[14] E. J. Small, P. F. Schellhammer, C. S. Higano et al., "Placebocontrolled phase III trial of immunologic therapy with sipuleucel-T (APC8015) in patients with metastatic, asymptomatic hormone refractory prostate cancer," Journal of Clinical Oncology, vol. 24, no. 19, pp. 3089-3094, 2006.
[15] F. S. Hodi, S. J. O’Day, D. F. McDermott et al., "Improved survival with ipilimumab in patients with metastatic melanoma," The New England Journal of Medicine, vol. 363, no. 8, pp. 711723, 2010.

[16] R. Yamanaka, "Cell- and peptide-based immunotherapeutic approaches for glioma," Trends in Molecular Medicine, vol. 14, no. 5, pp. 228-235, 2008.

[17] M. C. Kuppner, M. F. Hamou, and N. de Tribolet, "Immunohistological and functional analyses of lymphoid infiltrates in human glioblastomas," Cancer Research, vol. 48, no. 23, pp. 6926-6932, 1988.

[18] D. A. Mitchell, P. E. Fecci, and J. H. Sampson, "Immunotherapy of malignant brain tumors," Immunological Reviews, vol. 222, no. 1, pp. 70-100, 2008.

[19] B. Badie and J. Schartner, "Role of microglia in glioma biology," Microscopy Research and Technique, vol. 54, no. 2, pp. 106-113, 2001.

[20] A. M. Kostianovsky, L. M. Maier, R. C. Anderson, J. N. Bruce, and D. E. Anderson, "Astrocytic regulation of human monocytic/microglial activation," Journal of Immunology, vol. 181, no. 8, pp. 5425-5432, 2008.

[21] B. C. Kennedy, L. M. Maie, R. D. 'Amico, ” et al., "Dynamics of central and peripheral immunomodulation in a murine glioma model," BMC Immunology, vol. 10, article 11, 2009.

[22] D. S. Markovic, R. Glass, M. Synowitz, N. van Rooijen, and H. Kettenmann, "Microglia stimulate the invasiveness of glioma cells by increasing the activity of metalloprotease-2," Journal of Neuropathology and Experimental Neurology, vol. 64, no. 9, pp. 754-762, 2005.

[23] J. J. Watters, J. M. Schartner, and B. Badie, "Microglia function in brain tumors," Journal of Neuroscience Research, vol. 81, no. 3, pp. 447-455, 2005.

[24] H. Zhai, F. L. Heppner, and S. E. Tsirka, "Microglia/macrophages promote glioma progression," Glia, vol. 59, no. 3, pp. 472485, 2011.

[25] G. C. Daginakatte and D. H. Gutmann, "Neurofibromatosis1 (Nf1) heterozygous brain microglia elaborate paracrine factors that promote Nf1-deficient astrocyte and glioma growth," Human Molecular Genetics, vol. 16, no. 9, pp. 1098-1112, 2007.

[26] A. Wesolowska, A. Kwiatkowska, L. Slomnicki et al., "Microgliaderived TGF- $\beta$ as an important regulator of glioblastoma invasion-an inhibition of TGF- $\beta$-dependent effects by shRNA against human TGF- $\beta$ type II receptor," Oncogene, vol. 27, no. 7, pp. 918-930, 2008.

[27] S. Wagner, S. Czub, M. Greif et al., "Microglial/macrophage expression of interleukin 10 in human glioblastomas," International Journal of Cancer, vol. 82, no. 1, pp. 12-16, 1999.

[28] W. Penfield, "Microglia and the process of phagocytosis in gliomas," The American Journal of Pathology, vol. 1, no. 1, pp. 77-97, 1925.

[29] W. Li and M. B. Graeber, "The molecular profile of microglia under the influence of glioma," Neuro-Oncology, vol. 14, no. 8, pp. 958-978, 2012.

[30] J. MacMicking, Q. W. Xie, and C. Nathan, "Nitric oxide and macrophage function," Annual Review of Immunology, vol. 15, pp. 323-350, 1997.

[31] U. Boehm, T. Klamp, M. Groot, and J. C. Howard, "Cellular responses to interferon-gamma.," Annual Review of Immunology, vol. 15, pp. 749-795, 1997.

[32] S. F. Hussain, D. Yang, D. Suki, E. Grimm, and A. B. Heimberger, "Innate immune functions of microglia isolated from human 
glioma patients," Journal of Translational Medicine, vol. 4, article 15, 2006.

[33] P. Filipazzi, V. Huber, and L. Rivoltini, "Phenotype, function and clinical implications of myeloid-derived suppressor cells in cancer patients," Cancer Immunology, Immunotherapy, vol. 61, no. 2, pp. 255-263, 2012.

[34] C. Huettner, S. Czub, S. Kerkau, W. Roggendorf, and J. C. Tonn, "Interleukin 10 is expressed in human gliomas in vivo and increases glioma cell proliferation and motility in vitro," Anticancer Research, vol. 17, no. 5 A, pp. 3217-3224, 1997.

[35] I. F. Parney, "Basic concepts in glioma immunology," Advances in Experimental Medicine and Biology, vol. 746, pp. 42-52, 2012.

[36] D. Hanahan and R. A. Weinberg, "Hallmarks of cancer: the next generation," Cell, vol. 144, no. 5, pp. 646-674, 2011.

[37] E. A. Ling and W. C. Wong, "The origin and nature of ramified and amoeboid microglia: a historical review and current concepts," Glia, vol. 7, no. 1, pp. 9-18, 1993.

[38] F. Ginhoux, M. Greter, M. Leboeuf et al., "Fate mapping analysis reveals that adult microglia derive from primitive macrophages," Science, vol. 330, no. 6005, pp. 841-845, 2010.

[39] T. Morioka, T. Baba, K. L. Black, and W. J. Streit, "Inflammatory cell infiltrates vary in experimental primary and metastatic brain tumors," Neurosurgery, vol. 30, no. 6, pp. 891-896, 1992.

[40] R. A. Morantz, G. W. Wood, M. Foster, M. Clark, and K. Gollahon, "Macrophages in experimental and human brain tumorspart 2: studies of the macrophage content of human brain tumors," Journal of Neurosurgery, vol. 50, no. 3, pp. 305-311, 1979.

[41] K. L. Lambertsen, B. H. Clausen, A. A. Babcock et al., "Microglia protect neurons against ischemia by synthesis of tumor necrosis factor," Journal of Neuroscience, vol. 29, no. 5, pp. 1319-1330, 2009.

[42] J. Priller, A. Flügel, T. Wehner et al., “Targeting gene-modified hematopoietic cells to the central nervous system: use of green fluorescent protein uncovers microglial engraftment," Nature Medicine, vol. 7, no. 12, pp. 1356-1361, 2001.

[43] A. Flgel, M. Bradl, G. W. Kreutzberg, and M. B. Graeber, “Transformation of donor-derived bone marrow precursors into host microglia during autoimmune CNS inflammation and during the retrograde response to axotomy," Journal of Neuroscience Research, vol. 66, no. 1, pp. 74-82, 2001.

[44] M. Rodriguez, L. Alvarez-Erviti, F. J. Blesa et al., "Bone-marrowderived cell differentiation into microglia: a study in a progressive mouse model of Parkinson's disease," Neurobiology of Disease, vol. 28, no. 3, pp. 316-325, 2007.

[45] A. R. Simard, D. Soulet, G. Gowing, J. P. Julien, and S. Rivest, "Bone marrow-derived microglia play a critical role in restricting senile plaque formation in Alzheimer's disease," Neuron, vol. 49, no. 4, pp. 489-502, 2006.

[46] W. AlShakweer, Y. Alwelaie, A. M. Mankung, and M. B. Graeber, "Bone marrow-derived microglia in pilocytic astrocytoma," Frontiers in Bioscience (Elite Edition), vol. 3, pp. 371-379, 2011.

[47] B. Badie and J. M. Schartner, "Flow cytometric characterization of tumor-associated macrophages in experimental gliomas," Neurosurgery, vol. 46, no. 4, pp. 957-962, 2000.

[48] D. Germain and D. A. Frank, "Targeting the cytoplasmic and nuclear functions of signal transducers and activators of transcription 3 for cancer therapy," Clinical Cancer Research, vol. 13, no. 19, pp. 5665-5669, 2007.

[49] J. C. Rodrigues, G. C. Gonzalez, L. Zhang et al., "Normal human monocytes exposed to glioma cells acquire myeloid-derived suppressor cell-like properties," Neuro-Oncology, vol. 12, no. 4, pp. 351-365, 2010.

[50] J. M. Schartner, A. R. Hagar, M. van Handel, L. Zhang, N. Nadkarni, and B. Badie, "Impaired capacity for upregulation of MHC class II in tumor-associated microglia," Glia, vol. 51, no. 4, pp. 279-285, 2005.

[51] T. Hirano, K. Ishihara, and M. Hibi, "Roles of STAT3 in mediating the cell growth, differentiation and survival signals relayed through the IL-6 family of cytokine receptors," Oncogene, vol. 19, no. 21, pp. 2548-2556, 2000.

[52] E. C. Brantley and E. N. Benveniste, "Signal transducer and activator of transcription-3: a molecular hub for signaling pathways in gliomas," Molecular Cancer Research, vol. 6, no. 5, pp. 675684,2008 .

[53] T. Jansen, B. Tyler, J. L. Mankowski et al., "FasL gene knockdown therapy enhances the antiglioma immune response," Neuro-Oncology, vol. 12, no. 5, pp. 482-489, 2010.

[54] V. V. Didenko, H. N. Ngo, C. Minchew, and D. S. Baskin, "Apoptosis of $\mathrm{T}$ lymphocytes invading glioblastomas multiforme: a possible tumor defense mechanism," Journal of Neurosurgery, vol. 96, no. 3, pp. 580-584, 2002.

[55] A. L. Ford, E. Foulcher, F. A. Lemckert, and J. D. Sedgwick, "Microglia induce CD4 T lymphocyte final effector function and death," Journal of Experimental Medicine, vol. 184, no. 5, pp. 1737-1745, 1996.

[56] A. L. Ford, E. Foulcher, F. A. Lemckert, and J. D. Sedgwick, "Microglia induce CD4T lymphocyte final effector function and death," Journal of Experimental Medicine, vol. 184, no. 5, pp. 1737-1745, 1996.

[57] B. Badie, J. Schartner, S. Prabakaran, J. Paul, and J. Vorpahl, "Expression of Fas ligand by microglia: possible role in glioma immune evasion," Journal of Neuroimmunology, vol. 120, no. 1-2, pp. 19-24, 2001.

[58] S. Wintterle, B. Schreiner, M. Mitsdoerffer et al., "Expression of the B7-related molecule B7-H1 by glioma cells: a potential mechanism of immune paralysis," Cancer Research, vol. 63, no. 21, pp. 7462-7467, 2003.

[59] A. T. Parsa, J. S. Waldron, A. Panner et al., "Loss of tumor suppressor PTEN function increases B7-H1 expression and immunoresistance in glioma," Nature Medicine, vol. 13, no. 1, pp. 84-88, 2007.

[60] V. Samuels, J. M. Barrett, S. Bockman, C. G. Pantazis, and M. B. Allen, "Immunocytochemical study of transforming growth factor expression in benign and malignant gliomas," The American Journal of Pathology, vol. 134, no. 4, pp. 895-902, 1989.

[61] B. Badie, J. Schartner, J. Klaver, and J. Vorpahl, "In vitro modulation of microglia motility by glioma cells is mediated by hepatocyte growth factor/scatter factor," Neurosurgery, vol. 44, no. 5, pp. 1077-1083, 1999.

[62] J. C. Tsai, C. K. Goldman, and G. Y. Gillespie, "Vascular endothelial growth factor in human glioma cell lines: induced secretion by EGF, PDGF-BB, and bFGF," Journal of Neurosurgery, vol. 82, no. 5, pp. 864-873, 1995.

[63] C. Kjellman, S. P. Olofsson, O. Hansson et al., "Expression of TGF- $\beta$ isoforms, TGF- $\beta$ receptors, and SMAD molecules at different stages of human glioma," International Journal of Cancer, vol. 89, no. 3, pp. 251-258, 2000.

[64] S. Bodmer, K. Strommer, K. Frei et al., "Immunosuppression and transforming growth factor- $\beta$ in glioblastoma. Preferential production of transforming growth factor- $\beta 2$," Journal of Immunology, vol. 143, no. 10, pp. 3222-3229, 1989. 
[65] A. Wu, J. Wei, L. Y. Kong et al., "Glioma cancer stem cells induce immunosuppressive macrophages/microglia," Neuro-Oncology, vol. 12, no. 11, pp. 1113-1125, 2010.

[66] S. Koochekpour, A. Merzak, and G. J. Pilkington, "Vascular endothelial growth factor production is stimulated by gangliosides and TGF- $\beta$ isoforms in human glioma cells in vitro," Cancer Letters, vol. 102, no. 1-2, pp. 209-215, 1996.

[67] W. Wick, M. Platten, and M. Weller, "Glioma cell invasion: regulation of metalloproteinase activity by TGF- $\beta$," Journal of Neuro-Oncology, vol. 53, no. 2, pp. 177-185, 2001.

[68] R. Kiefer, R. Gold, J. Gehrmann, D. Lindholm, H. Wekerle, and G. W. Kreutzberg, "Transforming growth factor $\beta$ expression in reactive spinal cord microglia and meningeal inflammatory cells during experimental allergic neuritis," Journal of Neuroscience Research, vol. 36, no. 4, pp. 391-398, 1993.

[69] D. Lindholm, E. Castren, R. Kiefer, F. Zafra, and H. Thoenen, "Transforming growth factor- $\beta 1$ in the rat brain: increase after injury and inhibition of astrocyte proliferation," Journal of Cell Biology, vol. 117, no. 2, pp. 395-400, 1992.

[70] R. Kiefer, M. L. Supler, K. V. Toyka, and W. J. Streit, "In situ detection of transforming growth factor- $\beta$ mRNA in experimental rat glioma and reactive glial cells," Neuroscience Letters, vol. 166, no. 2, pp. 161-164, 1994.

[71] E. A. Maher, F. B. Furnari, R. M. Bachoo et al., "Malignant glioma: genetics and biology of a grave matter," Genes and Development, vol. 15, no. 11, pp. 1311-1333, 2001.

[72] C. Nolte, F. Kirchhoff, and H. Kettenmann, "Epidermal growth factor is a motility factor for microglial cells in vitro: evidence for EGF receptor expression," European Journal of Neuroscience, vol. 9, no. 8, pp. 1690-1698, 1997.

[73] S. J. Coniglio, E. Eugenin, K. Dobrenis et al., "Microglial stimulation of glioblastoma invasion involves EGFR and CSF-1R signaling," Molecular Medicine, vol. 18, pp. 519-527, 2012.

[74] S. Koochekpour, M. Jeffers, S. Rulong et al., "Met and hepatocyte growth factor/scatter factor expression in human gliomas," Cancer Research, vol. 57, no. 23, pp. 5391-5398, 1997.

[75] P. Kunkel, S. Müller, P. Schirmacher et al., "Expression and localization of scatter factor/hepatocyte growth factor in human astrocytomas," Neuro-Oncology, vol. 3, no. 2, pp. 82-88, 2001.

[76] J. Laterra, M. Nam, E. Rosen et al., "Scatter factor/hepatocyte growth factor gene transfer enhances glioma growth and angiogenesis in vivo," Laboratory Investigation, vol. 76, no. 4, pp. 565577, 1997.

[77] C. Eckerich, S. Zapf, R. Fillbrandt, S. Loges, M. Westphal, and K. Lamszus, "Hypoxia can induce c-Met expression in glioma cells and enhance SF/HGF-induced cell migration," International Journal of Cancer, vol. 121, no. 2, pp. 276-283, 2007.

[78] C. Sheng-Hua, M. Yan-Bin, Z. Zhi-An et al., "Radiationenhanced hepatocyte growth factor secretion in malignant glioma cell lines," Surgical Neurology, vol. 68, no. 6, pp. 610-613, 2007.

[79] A. Waziri, B. Killory, A. T. Ogden III et al., "Preferential in situ CD4+CD56+ T cell activation and expansion within human glioblastoma," Journal of Immunology, vol. 180, no. 11, pp. 76737680, 2008.

[80] A. El Andaloussi and M. S. Lesniak, "An increase in CD4+ CD25+FOXP3+ regulatory $\mathrm{T}$ cells in tumor-infiltrating lymphocytes of human glioblastoma multiforme," Neuro-Oncology, vol. 8, no. 3, pp. 234-243, 2006.

[81] P. E. Fecci, D. A. Mitchell, J. F. Whitesides et al., "Increased regulatory T-cell fraction amidst a diminished CD4 compartment explains cellular immune defects in patients with malignant glioma," Cancer Research, vol. 66, no. 6, pp. 3294-3302, 2006.

[82] H. Ohgaki and P. Kleihues, "The definition of primary and secondary glioblastoma," Clinical Cancer Research, vol. 19, no. 4, pp. 764-772, 2013.

[83] M. Mizoguchi, R. A. Betensky, T. T. Batchelor, D. C. Bernay, D. N. Louis, and C. L. Nutt, "Activation of STAT3, MAPK, and AKT in malignant astrocytic gliomas: correlation with EGFR status, tumor grade, and survival," Journal of Neuropathology and Experimental Neurology, vol. 65, no. 12, pp. 1181-1188, 2006.

[84] L. Zhang, D. Alizadeh, M. van Handel, M. Kortylewski, H. Yu, and B. Badie, "Stat3 inhibition activates tumor macrophages and abrogates glioma growth in mice," Glia, vol. 57, no. 13, pp. 1458$1467,2009$.

[85] L. K. Schaefer, D. G. Menter, and T. S. Schaefer, "Activation of Stat 3 and Stat1 DNA binding and transcriptional activity in human brain tumour cell lines by gp130 cytokines," Cellular Signalling, vol. 12, no. 3, pp. 143-151, 2000.

[86] M. Kortylewski, M. Kujawski, T. Wang et al., "Inhibiting Stat3 signaling in the hematopoietic system elicits multicomponent antitumor immunity," Nature Medicine, vol. 11, no. 12, pp. 13141321, 2005. 


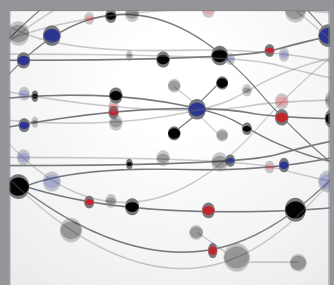

The Scientific World Journal
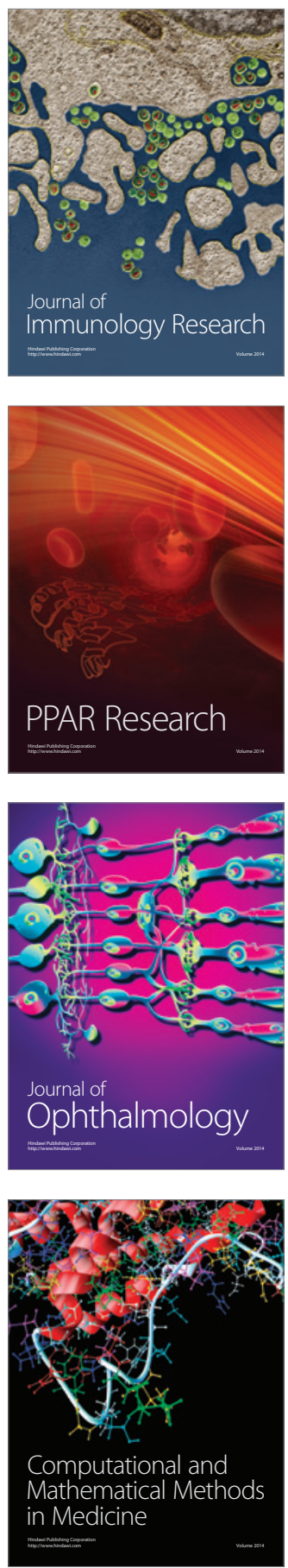

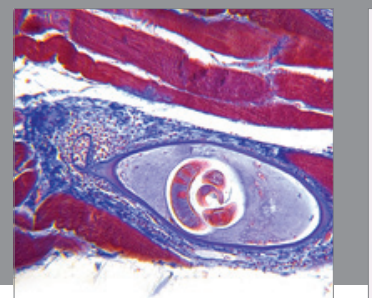

Gastroenterology

Research and Practice
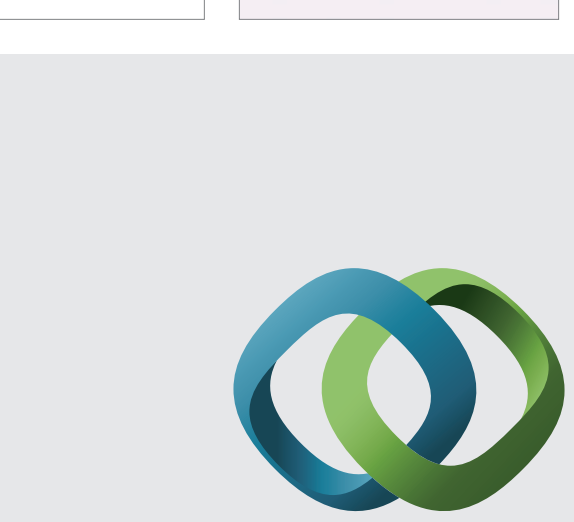

\section{Hindawi}

Submit your manuscripts at

http://www.hindawi.com
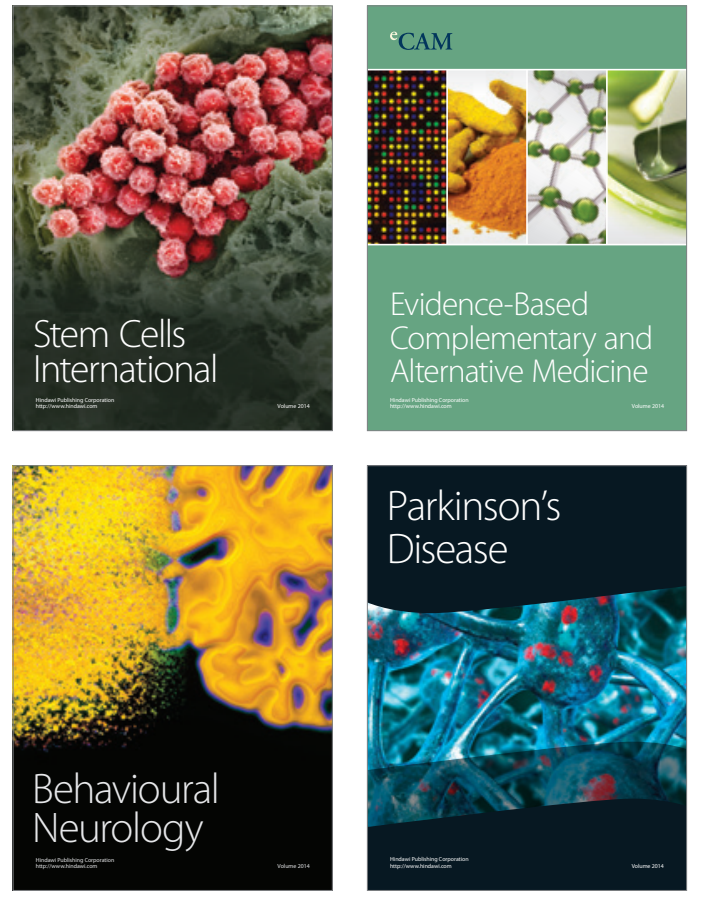
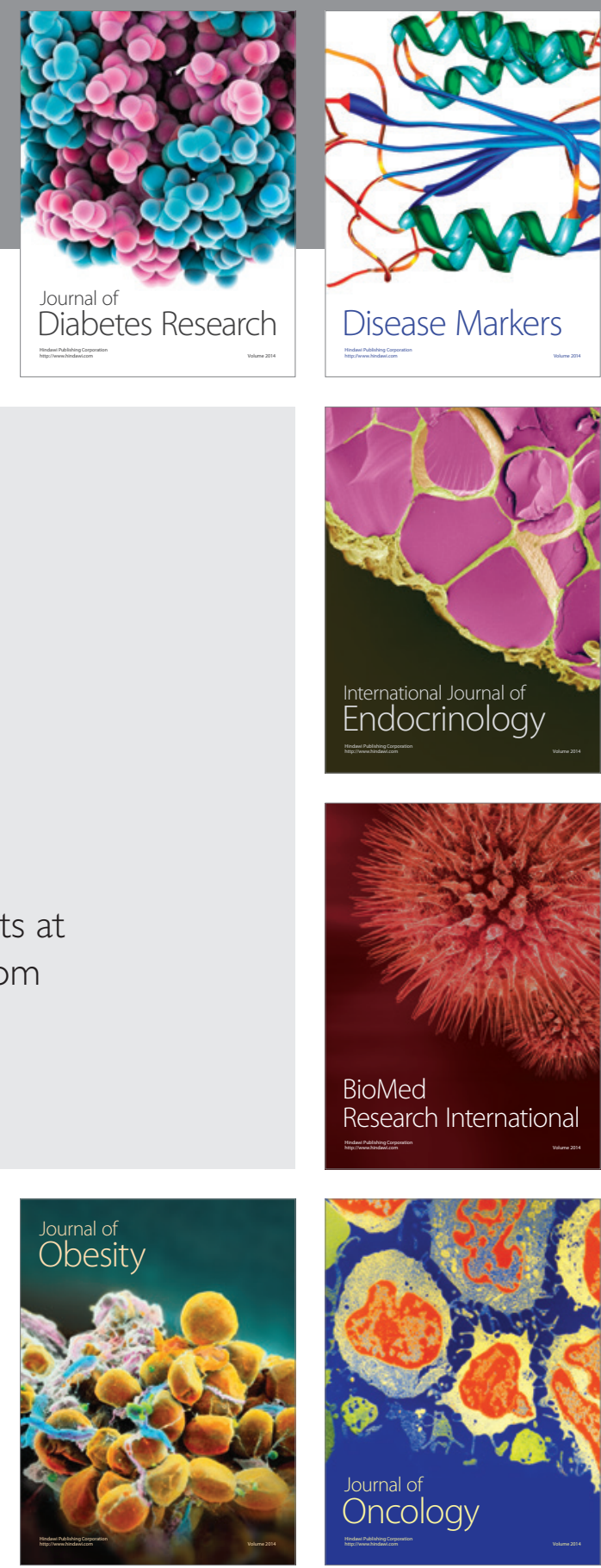

Disease Markers
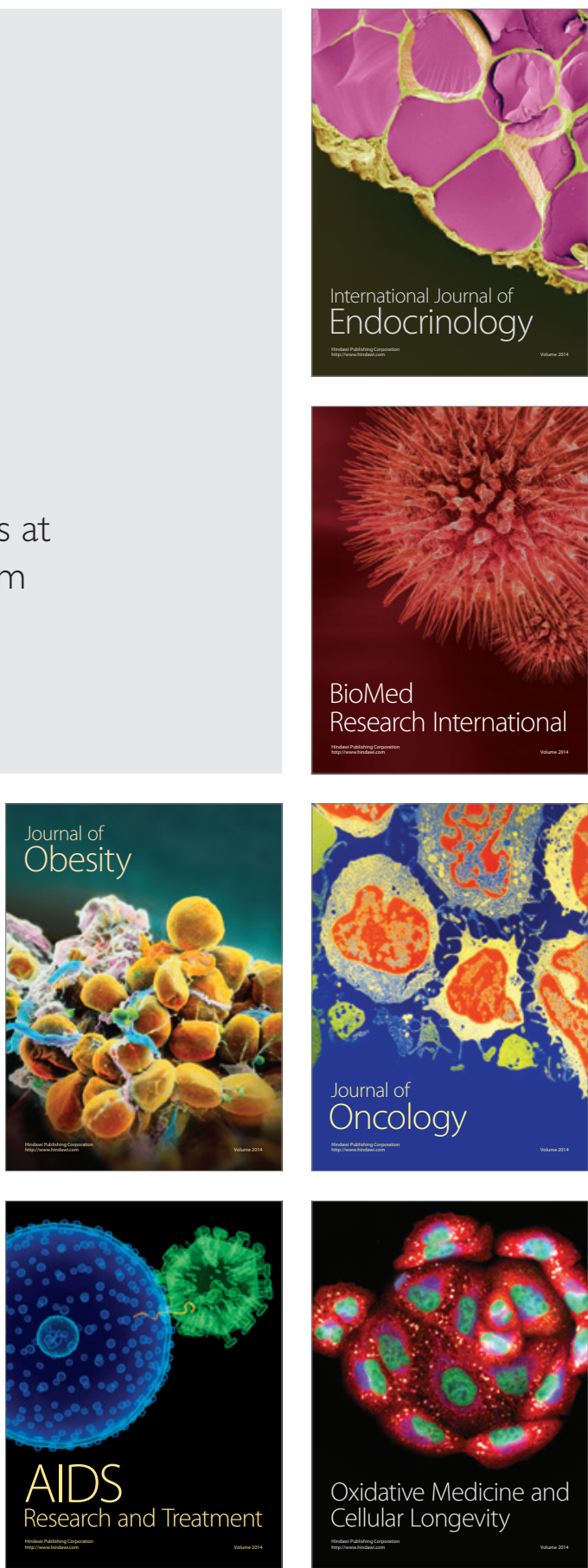